\title{
Introduction to Pediatric Congenital Heart Disease
}

\section{Gautam Singh, MD}

\section{Address}

Washington University School of Medicine, St. Louis, M0, USA

Email: Singh_G@kids.wustl.edu

Published online: 5 0ctober 2014

(C) Springer Science+Business Media New York 2014

This article is part of the Topical Collection on Pediatric Congenital Heart Disease

The current issue is dedicated to childhood obesity. Childhood obesity is now a global epidemic. Obese adolescents have an increased risk of becoming obese adults with higher morbidity and mortality rates from atherosclerotic cardiovascular disease than those adults who only become obese in adulthood. This issue is focused on the current state of knowledge about the impact of childhood obesity on cardiovascular structure and function, cardiac autonomic modulation, insulin resistance, and the associated cardiometabolic spectrum, with an emphasis on adaptive and maladaptive changes to understand the genesis of overt cardiovascular disease. Articles also highlight different management approaches to childhood obesity including lifestyle intervention, multi-component behavioral intervention with a focus on family-based behavioral intervention, immersion treatment, and pharmacologic and surgical management. We hope that readers will benefit from the scholarly articles in the management of childhood obesity in their practice. 